\title{
Quantum theory of incompatible observations
}

\author{
Z. Hradil and J. Summhammer \\ Atominstitut der Österreichischen Universitäten, \\ Stadionallee 2, \\ A-1020 Wien, Austria
}

(June 24, 2021)

\begin{abstract}
Maximum likelihood principle is shown to be the best measure for relating the experimental data with the predictions of quantum theory.
\end{abstract}

Quantum theory describes events on the most fundamental level currently available. The synthesis of information from mutually incompatible quantum measurements plays the key role in testing the structure of the theory. The purpose of this Letter is to show a unique relationship between quantum theory and the mathematical statistics used to obtain optimal information from incompatible observations: Quantum theory prefers the relative entropy (maximum likelihood principle) as the proper measure for evaluation of the distance between measured data and probabilities defined by quantum theory.

In the standard textbooks [1], a quantum measurement is represented by a hermitian operator $\hat{A}$, whose spectrum determines the possible results of the measurement

$$
\hat{A}|a\rangle=a|a\rangle
$$

In the following, the Dirac notation will be used and for the sake of simplicity a discrete spectrum will be assumed. Eigenstates are orthogonal $\left\langle a \mid a^{\prime}\right\rangle=\delta_{a a^{\prime}}$ and the corresponding projectors provide the closure relation

$$
\sum_{a}|a\rangle\langle a|=\hat{1}
$$

Projectors predict the probability for detecting a particular value of the q-variable $a$ represented by the operator $\hat{A}$ as $p_{a}=\langle a|\rho| a\rangle$, provided that the system has been prepared in a quantum state $\rho$. This mathematical picture corresponds to the experimental reality in the following sense: When the measurement represented by the operator $\hat{A}$ is repeated $\mathrm{N}$ times on identical copies of the system, the number a particular output $a$ is collected $N_{a}$ times. The relative frequencies $f_{a}=\frac{N_{a}}{N}$ will sample the true probability as $f_{a} \rightarrow p_{a}$ fluctuating around them. The exact values are reproduced only in the asymptotical limit $N \rightarrow \infty$. Experimentalist's knowledge may be expressed in the form of a diagonal density matrix

$$
\hat{\rho}_{\text {est }}=\sum_{a} f_{a}|a\rangle\langle a|,
$$

provided that error bars of the order $1 / N$ are associated with the sampled relative frequencies. This should be understood as mere rewriting of the experimental data $\left\{N, N_{a}\right\}$. Similar knowledge may be obtained by observations, which can be parameterized by operators diagonal in the $|a\rangle$ basis, i.e. by operators commuting with operator $\hat{A}$. But the possible measurement of non-commuting operators yields new information, which cannot be derived from the measurement of $\hat{A}$.

From this viewpoint it seems to be advantageous to consider the sequential synthesis of various noncommuting observables. In this case several operators $\hat{A}_{j}, j=1,2, \ldots$ will be measured by probing of the system $N$ times together. Now, one expects to gain more than just the knowledge of the diagonal elements of the density matrix in some a priori given basis. This sequential measurement of non-commuting observables should be distinguished from the similar problem of approximate simultaneous measurement of non-commuting observables 22. As in the case of the measurement of a hermitian operators, the result of sequential measurements of noncommuting operators may be represented by a series of projectors $\left|y_{i}\right\rangle\left\langle y_{i}\right|$. This should be accompanied by relative frequencies $f_{i}$ indicating how many times a particular output $i$ has been registered, $\sum_{i} f_{i}=1$. Various states need not be orthogonal $\left\langle y_{i} \mid y_{j}\right\rangle \neq \delta_{i j}$, in contrast to the previous case of a hermitian operator. However, this substantial difference has its deep consequences. The result of the measurement cannot be meaningfully represented in the same manner as previously. For example, direct linking of probabilities with relative frequencies used in standard reconstructions [3,4] $\rho_{i i}=f_{i}, \rho_{i i}=\left\langle y_{i}|\hat{\rho}| y_{i}\right\rangle$, may appear as inconsistent, since the system of linear equation is overdetermined, in general.

A novel approach will be suggested here. Let us assume the existence of a quantum measure $F\left(\rho_{i i} \mid f_{i}\right)$, which parameterizes the distance between measured data and probabilities predicted by quantum theory. We will search for the state(s) located in the closest neighborhood of the data. A general state may be parameterized in its diagonal basis as

$$
\hat{\rho}=\sum_{i} r_{i}\left|\varphi_{i}\right\rangle\left\langle\varphi_{i}\right|
$$

The equation for the extremal states may be found analogously to the treatment developed in [5] for maximum likelihood estimation as 


$$
\sum_{i} \frac{\partial F}{\partial \rho_{i i}}\left|y_{i}\right\rangle\left\langle y_{i}\right| \hat{\rho}=\lambda \hat{\rho},
$$

where $\lambda$ is a Lagrange multiplier. The normalization condition $\operatorname{Tr} \hat{\rho}=1$ sets its value to

$$
\lambda=\sum_{i} \frac{\partial F}{\partial \rho_{i i}} \rho_{i i} .
$$

Any composed function $G\left(F\left(\rho_{i i} \mid f_{i}\right)\right)$ fulfills the same extremal equation (5) with the Lagrange multiplier rescaled as $\lambda \frac{d G}{d F}$. Without loss of generality it is therefore enough to consider the normalization condition $\lambda=1$.

The extremal equation (5) has the form of a decomposition of the identity operator on the subspace, where the density matrix is defined by

$$
\sum_{i} \frac{\partial F}{\partial \rho_{i i}}\left|y_{i}\right\rangle\left\langle y_{i}\right|=\hat{1}_{\rho} .
$$

This resembles the definition of a probability-valued operator measure (POVM) characterizing a generalized measurement [6]. To link the above extremalization with quantum theory, let us postulate the natural condition for the quantum expectation value

$$
\operatorname{Tr}\left(\frac{\partial F}{\partial \rho_{i i}}\left|y_{i}\right\rangle\left\langle y_{i}\right| \hat{\rho}\right)=f_{i} .
$$

This assumption seems to be reasonable. The synthesis of sequential non-compatible observations may be regarded as a new measurement scheme, namely the measurement of the quantum state.

The quantum measure $F$ then fulfills the differential equation

$$
\frac{\partial F}{\partial \rho_{i i}} \rho_{i i}=f_{i} .
$$

and singles out the solution in the form

$$
F\left(\rho_{i i} \mid f_{i}\right)=\sum_{i} f_{i} \ln \rho_{i i}
$$

This is nothing else than the log likelihood or KullbackLeibler relative information [7]. Formal requirements of quantum theory, namely the interpretation of the extremal equation as a POVM, result in the concept of maximum likelihood in mathematical statistics. The analogy between the standard quantum measurement associated with a single hermitian operator, and a series of sequential measurements associated with many non-commuting operators is apparent now. The former determines the diagonal elements in the basis of orthonormal eigenvectors, whereas the latter estimates not only the diagonal elements, but the diagonalizing basis itself. This is the difference between measurement of quantum observable $\hat{A}$ and measurement of the quantum state. In this sense maximum likelihood estimation may be considered as a new quantum measurement. The observed quantum state is given by the solution of the nonlinear operator equation

$$
\sum_{i} \frac{f_{i}}{\rho_{i i}}\left|y_{i}\right\rangle\left\langle y_{i}\right| \hat{\rho}=\hat{\rho}
$$

which is, in fact, the completeness relation of a POVM with measured outputs $\left\{f_{i}\right\}$. Special cases of the solution (10) have been discussed recently for the phase [8], the diagonal elements of the density matrix [9] and the reconstruction of the $1 / 2$ spin state [10].

Quantum interpretation offers a new viewpoint on the maximum likelihood estimation. This method is customarily considered as just one of many estimation methods, unfortunately one of the most complicated ones. It is often considered as a subjective method, since likelihood quantifies the degree of belief in a certain hypothesis. Any physicist, an experimentalist above all, would perhaps use as the first choice the least-squares method for fitting theory and data [4]. Let us evaluate this as an illustrative counterexample. In this case $F\left(\rho_{i i} \mid f_{i}\right)=\sum_{i}\left(\rho_{i i}-f_{i}\right)^{2}$ and the extremal equation reads

$$
\begin{array}{r}
2 \sum_{i}\left(\rho_{i i}-f_{i}\right)\left|y_{i}\right\rangle\left\langle y_{i}\right| \hat{\rho}=\lambda \hat{\rho}, \\
\lambda=2 \sum_{i}\left(\rho_{i i}-f_{i}\right) \rho_{i i} .
\end{array}
$$

Equation (11) may be again interpreted as a completeness relation for the POVM

$$
\hat{E}_{i}=2 \frac{\left(\rho_{i i}-f_{i}\right)}{\lambda}\left|y_{i}\right\rangle\left\langle y_{i}\right| .
$$

However the expectation value is a rather complicated implicit function of the measured data, since

$$
\operatorname{Tr}\left(\hat{\rho} \hat{E}_{i}\right)=2 \frac{\left(\rho_{i i}-f_{i}\right) \rho_{i i}}{\lambda} .
$$

It does not mean that the least-squares method is incorrect, it only means that such fitting does not reveal the structure of quantum measurement. In this sense the maximum likelihood seems to be unique and exceptional.

There are several fundamental consequences of this result. According to Fisher's theorem [11, maximum likelihood estimation is unbiased and achieves the CramérRao bound asymptotically for large $N \rightarrow \infty$. As demonstrated here, for any finite $N$ maximum likelihood may be interpreted as a quantum measurement. When seen this way, bias and the noise above the Cramér-Rao bound seem to be unpleasant but natural properties of quantum systems. Maximum likelihood may set new bounds on distinguishability related currently to the Fisher information [12]. Fisher information corresponds to the Riemannian distinguishability metrics and may be naturally interpreted as the distance in the Hilbert space. Besides this, fundamental equations of quantum theory such as 
Schrödinger, Klein-Gordon, Pauli etc. and other physical laws may be derived from the principle of minimum Fisher information 113,14. Since Fisher information only approximates the behaviour of likelihood in asymptotical limit, all these features seem to be involved also in the maximum likelihood principle. However, the latter one is obviously stronger since, as shown here, only the maximum likelihood is able to reproduce the structure of quantum measurement for finite observations.

Notice also that maximum likelihood generalizes the notion of POVM in the following sense. Actual measurements may be (and usually always are) incomplete. However, the synthesis of any incomplete measurements, namely of the original observations represented here by $\left|y_{i}\right\rangle\left\langle y_{i}\right|$, is complete in the subspace, where the resolution of the identity (6) holds. POVM and estimated quantum state are mutually connected in dependence on the type of measurement and on its results. In particular, it is not necessary to consider only the special scheme for quantum state observation as for example the mutually complementary eigenbases 15 .

We conclude with a remark that may shed light on why maximum likelihood is peculiar: Maximum likelihood is perhaps singled out by Nature, because the nonsymmetric fluctuations of data of multinomial distributions, which are the results of quantum measurements, are compensated, so to say, by an equally non-symmetric attribution of degrees of belief to the various test states. Maximum likelihood takes into account that, in finite observations, improbable events tend to appear more frequently than they should, and conversely, very probable events tend to appear somewhat less often.

This work was supported by TMR Network ERB FMRXCT 96-0057 "Perfect Crystal Neutron Optics" of the European Union and by the grants of Czech Ministry of Education VS 96028 and CEZ J14/98.

* Permanent address: Department of Optics, Palacký University, 17. listopadu 50, 77207 Olomouc, Czech Republic.

[1] J. J. Sakurai, Modern Quantum Mechanics, AddisonWesley, 1994.

[2] The approximate simultaneous measurement of noncommuting operators $[\hat{A}, \hat{B}] \neq 0$ can always be represented by measurement of commuting operators $\hat{\mathcal{A}}, \hat{\mathcal{B}}$ defined on the extended Hilbert space $\mathcal{H}=H_{s} \otimes H_{a}$, where $H_{s}, H_{a}$ is the space of original system and space of auxiliary field (ancilla), respectively.

[3] U. Leonhardt, Measuring of the Quantum State of Light, Cambridge Press, 1997.

[4] D.- G. Welsch, W. Vogel, T. Opatrný, "Homodyne Detection and Quantum State Reconstruction", in Progress in Optics, 39 , ed. E. Wolf.
[5] Z. Hradil, Phys. Rev. A 55, R1561 (1997); Z. Hradil, J. Summhammer, H. Rauch, Phys. Lett. A 261 (1999) 20.

[6] C. W. Helstrom, Quantum Detection and Estimation Theory, Academic Press, New York 1976.

[7] S. Kullback, R. A. Leibler, Ann. of Math. Stat. 22, 79 (1951). Notice the asymmetry between the arguments $f$ and $p$ in definition of Kullback-Leibler relative information $K(f / p)=\sum_{i} f_{i} \ln \left(f_{i} / p_{i}\right)$. In the paper of B. R. Frieden, in Maximum Entropy and Bayesian Methods in Inverse Problems, edited by C. R. Smith, W. T. Grandy Jr. (Reidel, Dordrecht 1985), p. 133 is the term KullbackLeibler norm used for opposite ordering of data and probabilities. The case discussed here is called generalized Burg principle.

[8] J. Řeháček, Z. Hradil, M. Zawisky, S. Pascazio, H. Rauch, J. Peřina, Phys. Rev. A 60, 4731999.

[9] Z. Hradil, R. Myška, acta phys. slov. 48, 199 (1998).

[10] Z. Hradil, J. Summhammer, G. Badurek, H. Rauch, Spin state reconstruction, unpublished.

[11] R. A. Fisher, Proc. Camb. Soc. 22, 700 (1925).

[12] W. K. Wootters, Phys. Rev. D 23, 357 (1981); S. L. Braunstein, C. M. Caves, Phys. Rev. Lett. 72, 3439 (1994).

[13] M. Reginatto, Phys. Rev. A 58, 1775 (1998); M. Reginatto, Phys. Lett. A 249, 355 (1998).

[14] B. Roy Frieden, Physics from Fisher Information, Cambridge Univ. Press, Cambridge 1999.

[15] W. K. Wootters, Found. Phys. 16, 391 (1986). 